\title{
A Quantitative LC-MS/MS Study of the Partitioning, Transport, and Fate of Pesticide Residues on Soil
}

\author{
Heather A. Gamble1, Donald S. Gamble² \\ ${ }^{1}$ Ionics Mass Spectrometry Group, Inc., Bolton, Canada \\ ${ }^{2}$ Department of Chemistry, St. Mary's University, Halifax, Canada \\ Email: heatherg@ionics.ca
}

Received 18 January 2016; accepted 23 February 2016; published 26 February 2016

Copyright (C) 2016 by authors and Scientific Research Publishing Inc.

This work is licensed under the Creative Commons Attribution International License (CC BY). http://creativecommons.org/licenses/by/4.0/

(c) (i) Open Access

\section{Abstract}

Titration of pesticides onto sorption sites can determine sorption capacities on soils. Previous studies have tracked the sorption capacities and detailed kinetics of the uptake of atrazine and its decomposition byproduct hydroxyatrazine on different soils, including measurements made using LC-MS/MS. These studies have now been extended to explore sorption-desorption equilibria for a mixture of pesticides from soil using LC-MS/MS. Desorption of sorbed pesticide residues has environmental regulatory implications for pesticide levels in runoff, or for longer term sequestration, partitioning, and transport. The uptake of pesticides by the soil at equilibrium was measured for a number of different concentrations, and sorption capacities were estimated. Pesticide-soil interaction studies were conducted by exposing standard stock solutions of pesticide mixtures to a characterized Nova Scotia soil. The mixture contained atrazine and dicamba. Initial aqueous mixture concentrations ranging from $5 \times 10^{-9}$ to $10^{-5} \mathrm{M}$ or greater were exposed to $25 \mathrm{mg}$ aliquots of soil and allowed to reach equilibrium. The total uptake of each pesticide was measured indirectly, by measuring the concentration remaining in solution using an IONICS $3 Q 120$ triple quadrupole mass spectrometer. These sorption capacities have been supplemented by studies examining equilibrium recovery rates from soil aliquots with different initial uptakes. This gives insight into the fraction of easily recoverable (reversibly sorbed) pesticides on the soil. Proper quantification of equilibrium constants and kinetic rate coefficients using high performance LC-MS/MS facilitates the construction of accurate, predictive models. Predictive kinetic models can successfully mimic the experimental results for solution concentration, labile sorption, and intra-particle diffusion, and could be used to guide regulatory practices.

\section{Keywords}

Pesticide-Soil Interactions, Sorption Kinetics, LC-MS/MS Fast Polarity Switching, Predictive Modeling 


\section{Introduction}

Current commercial agricultural practices involve the widespread use of pesticides applied to crops in order to maximize yields. The use of such pesticides facilitates the cost-efficient production of large quantities of high quality food suitable for widespread commercial distribution, feeds large numbers of people, and supports an essential sector in the world economy. However, these compounds are inherently toxic at some level, and due consideration should be given to the possible effects of releasing large quantities into the environment. Questions arise such as, where do these compounds end up, and in what form? Dissipation into rivers, lakes, or other waterways might reduce local concentrations of pesticide residues and their possible decomposition byproducts, but does not remove them from the environment on the whole. Instead, it creates potential issues of long range transport and bioaccumulation.

Environmental Law is concerned with chemical contamination in soil, water, and air, which in practice are interconnected. It includes legislation, regulatory decisions, contracts, litigation and potentially criminal charges. For soil and water this presents environmental forensic technology with serious challenges. A soil or aquatic sediment is the ultimate example of a physically and chemically irregular mixture. It is also dynamic. The basic science required to understand the interactions between contaminants and soils, at a fundamental level, as well as the transport of contaminants for example via surface or groundwater is now emerging. To get a complete picture, our understanding of interactions, and of transport, would ideally be combined. This would mean updating and integrating individual models, which are currently fairly modular. Opportunities for starting this integration are explored in this article. A properly integrated understanding of the fate of contaminants in the environment would be a valuable tool. It could be used to determine appropriate levels of concern, based on usage levels and local factors such as soil type, amount of precipitation, and proximity to local watersheds. It would also potentially be useful in suggesting control strategies for containment, treatment, or remediation.

Models which use fundamental kinetic rate coefficients to accurately predict the rate of uptake of specific pesticides, and their total sorption capacities, on recognized soils already exist and are well documented [1] [2]. They are based on the fundamental premise that sorption-desorption is a labile process that can be written as a reaction between the analyte $M$ (e.g. a pesticide) and reaction sites on the surface of the soil $(X)$ to produce occupied sorption sites $M X$ :

$$
M+X \stackrel{K}{\rightleftharpoons} M X .
$$

It is possible to experimentally determine kinetic rate coefficients for the sorption reactions of various pesticides on specific soils. If equilibrium can be attained, it is also possible to determine the uptake capacity $\theta$ of a particular soil-pesticide combination, based on the equilibrium condition

$$
K=\frac{\left[M_{1} X\right]}{\left[M_{1}\right][X]}
$$

Or equivalently,

$$
K=\frac{\theta_{1}}{M_{1}\left(\theta-\theta_{1}\right)}
$$

In the above equations, $M_{1}$ represents the solution equilibrium concentration of a specific pesticide; $\theta_{1}$ represents the equilibrium concentration $\left[M_{1} X\right]$ of occupied sorption sites; and $\theta-\theta_{1}$ gives the equilibrium concentration $[X]$ of unoccupied sites.

Sorption onto active surface sites begins immediately upon exposure of the soil to the pesticides. Intra-particle diffusion into pores also occurs, and is governed by its own kinetics. Diffusion both into and out of the pores tends to be slower. This creates two broad categories of adsorbed residues: Surface adsorbed, relatively labile residues, and more slowly released residues sequestered within the soil particles. After more prolonged exposure, decomposition products can emerge, potentially including unknown species. From an environmental perspective, these decomposition products can be either more or less benign than the parent compounds. Experimental techniques exist which can resolve these fractions [2]. Sorption site stoichiometry can be revealed by titrating the analyte onto the labile sorption sites. Kinetics curves can be constructed for each of the intraparticle-diffused, labile-sorbed and solution phase fractions.

Eleven user friendly spreadsheet models based on molecular level kinetics and mechanisms exist. These mod- 
els predict the uptake rates and sorption capacities of select pesticides on characterized soils, using the experimentally determined fundamental rate coefficients. Descriptions and testing have been published for three of them [3]-[6]. These $a b$ initio calculations based on rate coefficients from first principles are much more accurate for prediction and extrapolation than more empirically based distribution coefficients, in which the fundamental chemistry can be obscured. In fact, the shortcomings of these empirical models have been recognized for some time [1] [7] [8]. From the environmental and regulatory perspective, these kinetic models therefore represent an advance in techniques that can be used to accurately account for one part of the overall problem, namely details of how contaminants interact with soils, including issues such as uptake, chemical decomposition, short term release, and possible sequestration.

These kinetic models do not address issues of short or long range transport in the environment, for example tracing the movements of contaminated surface or ground waters as they move through local watersheds. These issues belong in the domains of soil science and hydrology engineering. Various hydrology models exist, for tracking and predicting the movement of water through local watersheds. Some are quite comprehensive and account for local geology and weather conditions. One such model is the Pesticide Root Zone Model for Ground Water (PRZM GW) model [9], which is designed to help users predict the effects of pesticide applications to fields. The model is very flexible and allows users to specify amounts of pesticides and a number of applications per year, then predict the dispersion based on hydrological and chemical processes.

Although comprehensive, and correct in its attempts to integrate hydrology effects and chemistry, this model makes use of empirical half-lives and distribution coefficients to predict chemical effects. As demonstrated, these predictions can be out by as much as an order of magnitude.

Environmental Forensic science faces a serious challenge. Two categories of predictive models presently exist. While the chemistry category of models described here correctly account for the chemical mechanisms, they do not account for the water transport of chemicals through soils. The other categories of models are the pesticide fate and transport models which are based on hydrology engineering. They have reasonable descriptions of the water transport of chemicals through soils and undertake to account for agronomy. Their attempts to describe chemical mechanisms are however totally unrealistic. The legally important examples are the various editions of the pesticide fate and transport model PRZM.gw. The Pest Management Regulatory Agency (Health Canada) and the U.S. EPA collaborate under the NAFTA agreement to harmonize pesticide regulations. When tested with experimental data, PRZM.gw is found to make predictions that are wrong by 1 to 3 orders of magnitude. If criminal charges or litigation reach the courts, environmental forensics will have real difficulties.

A desirable long term goal would be the integration of comprehensive hydrology and pesticide application and dispersion models such as PRZM GW, with the kinetics models which accurately predict the fate of pesticides interacting with soils using fundamental chemical processes and experimentally determined rate coefficients. To do so, it is necessary to conduct careful laboratory experiments using modern analytical techniques which can determine accurate rate coefficients and uptake capacities for a variety of pesticides and their possible decomposition products on different soil types and soil components. By using high performance LC-MS/MS, it is possible to rapidly obtain large quantities of good quality data for the uptake, partitioning, and release of a large number of pesticides by different soil types or components. We present here a snapshot of such experiments, using LC-MS/MS to measure uptake and recovery of atrazine and dicamba from a Nova Scotia soil. These experiments could easily be extended, and demonstrate the feasibility of obtaining the accurate, highly precise kinetic data needed to help create a comprehensive, chemically correct, integrated model.

\section{Methodology}

\subsection{Experimental Determination of Sorption Capacities}

Experiments were conducted to examine sorption capacities and release (desorption) properties of atrazine and dicamba on a sample of Nova Scotia soil. The soil sample was collected from the north shore of Nova Scotia, along the Northumberland Straight. This soil is characterized as sandy. X-Ray Fluorescence Spectroscopy revealed the primary components of the soil to be silica (74.23\%) and alumina (10.29\%), with traces of $\mathrm{Fe}_{2} \mathrm{O}_{3}$, $\mathrm{Na}_{2} \mathrm{O}, \mathrm{K}_{2} \mathrm{O}, \mathrm{MgO}$, and $\mathrm{TiO}_{2}$. This composition is very typical of the region. An electron micrograph (Figure 1) shows the irregular and potentially porous nature of the soil particles.

Atrazine (98.8\%), dicamba (99.4\%), and hydroxyatrazine (98.8\%) were acquired as pure solids from Sigma Aldrich. The solids were dissolved in HPLC grade water to generate a set of standard solutions with concentrations 


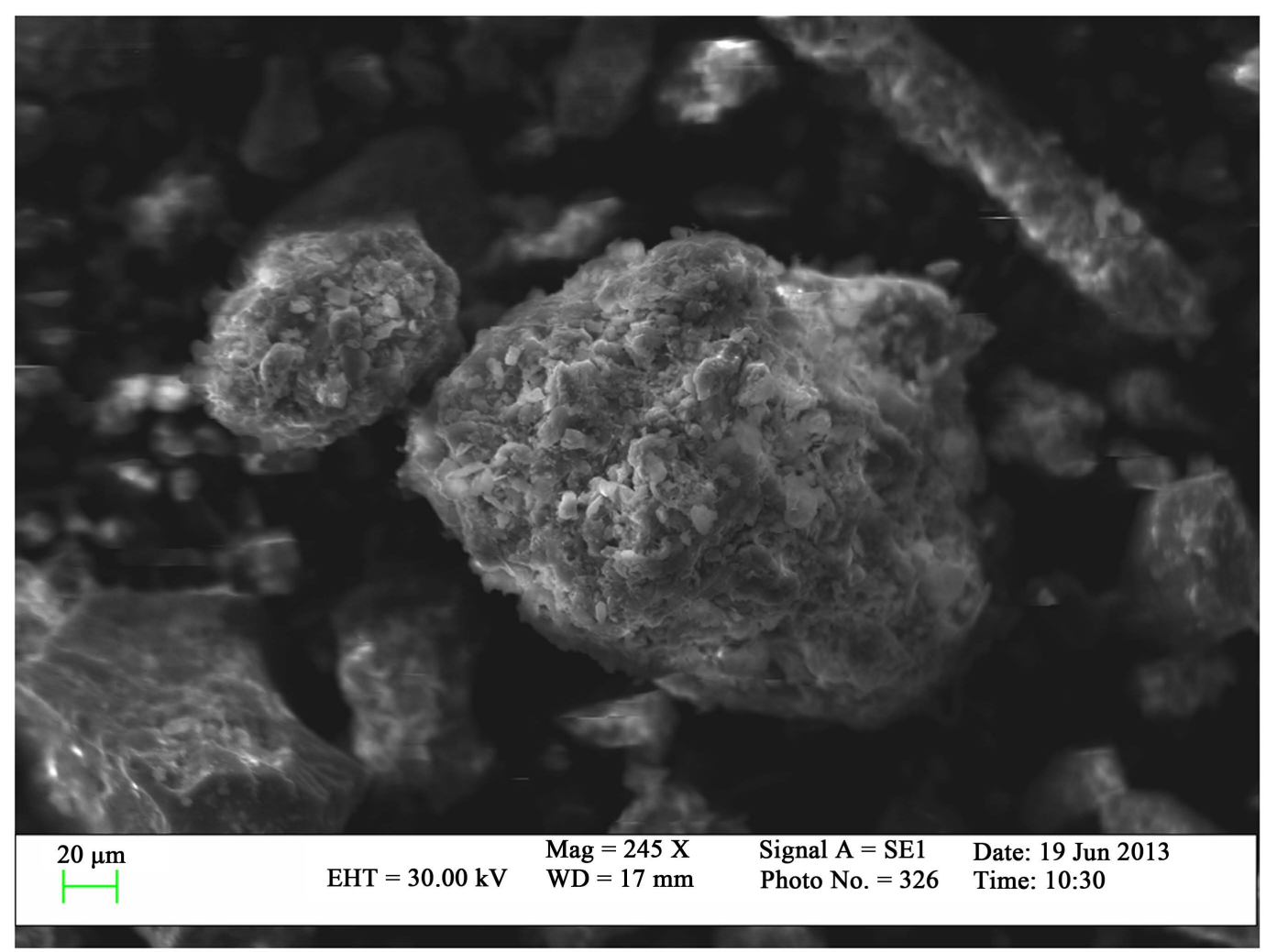

Figure 1. Electron micrograph of the Nova Scotia soil sample, showing surface and particle size irregularities.

ranging from $5 \times 10^{-5} \mathrm{M}$ to $5 \times 10^{-9} \mathrm{M}$ in each of atrazine and dicamba (1 - 10,000 ppb w/w). Sorption capacity studies were conducted by adding these standard solutions to $20 \mathrm{~mL}$ glass vials containing weighed, dried $25 \mathrm{mg}$ aliquots of soil. A total volume of $10 \mathrm{~mL}$ of stock solution was added to each vial, which was then sealed. The soil-pesticide systems were then left to equilibrate at room temperature. The vials were agitated briefly approximately once per day. After eight to twelve weeks, the uptake of pesticides by each soil aliquot was determined by measuring the concentrations remaining in solution.

Recovery of sorbed pesticide residues was explored by selecting some exposed aliquots of soil, air drying them, exposing them at room temperature to $3 \mathrm{~mL}$ of pure HPLC grade water in sealed $20 \mathrm{~mL}$ glass vials for 1 and 11 days, then measuring the concentrations of pesticides recovered from the soil. During the desorption process, the vials were once again agitated briefly approximately once per day.

Solution concentrations in general were determined using an IONICS 3Q 120 LC-MS/MS system consisting of two HPLC pumps, an autosampler, an oven, and a triple quadrupole mass spec detector with a dual probe ion source, HSID ${ }^{\mathrm{TM}}$ interface and unifield detector. Each injection volume was $15 \mu \mathrm{L}$. Good separation of atrazine, its decomposition byproduct hydroxyatrazine, and dicamba was achieved using a C18 column and an isocratic mixture of 50:50 organic to aqueous phase consisting of water/methanol, $0.1 \%$ formic acid and $50 \mathrm{mM}$ ammonium acetate with a total flow of $0.50 \mathrm{~mL} / \mathrm{min}$. The ion source of the mass spectrometer was operated in ESI mode. The source temperature was set at $350^{\circ} \mathrm{C}$ to accommodate dicamba, which decomposes at higher temperatures. As is customary the mass spectrometer was operated in Multiple Reactant Monitoring (MRM) mode, in which each resolving quadrupole is set to transmit a fixed mass, rather than scanning an entire peak or mass range. This reduces the scanning time and facilitates near-simultaneous acquisition of data from multiple parent/ fragment ion MRM pairs. Table 1 lists the MRM transitions and conditions used for atrazine, dicamba, and hydroxyatrazine. The highest mass fragments were used as quantifiers for atrazine and hydroxyatrazine $(\mathrm{m} / \mathrm{z}+216$ to +104 and $m / z+198$ to +154 respectively), while for dicamba the $m / z-219$ to -175 transition overwhelmingly dominated the spectrum.

Calibration curves were generated for each run, using standard pesticide mixtures which had been diluted using HPLC grade water which had previously been exposed to the soil, to reduce possible ion source matrix 
Table 1. MRM transitions and voltages used for atrazine, dicamba, and hydroxyatrazine.

\begin{tabular}{|c|c|c|c|c|c|}
\hline Compound & Solubility $\left(\mathrm{H}_{2} \mathrm{O}\right)$ & Melting Pt $\left({ }^{\circ} \mathrm{C}\right)$ & $\mathrm{EV}(\mathrm{V})$ & $\operatorname{MRM}(m / z)$ & CE (V) \\
\hline \multirow{3}{*}{$\begin{array}{l}\text { Atrazine } \\
\text { (ESI Pos) }\end{array}$} & \multirow{3}{*}{$7 \mathrm{mg} / 100 \mathrm{~mL}$} & \multirow{3}{*}{175} & \multirow{3}{*}{+70} & $+216 /+104$ & -40 \\
\hline & & & & $+216 /+132$ & -35 \\
\hline & & & & $+216 /+174$ & -25 \\
\hline \multirow[t]{2}{*}{$\begin{array}{l}\text { Dicamba } \\
\text { (ESI Neg) }\end{array}$} & \multirow[t]{2}{*}{$50 \mathrm{~g} / 100 \mathrm{~mL}$} & \multirow[t]{2}{*}{115} & \multirow[t]{2}{*}{-20} & $-219 /-175$ & +10 \\
\hline & & & & $+198 /+86$ & -35 \\
\hline \multirow[t]{2}{*}{$\begin{array}{l}\text { H-Atrazine } \\
\text { (ESI Pos) }\end{array}$} & \multirow[t]{2}{*}{$0.59 \mathrm{~g} / 100 \mathrm{~mL}$} & \multirow[t]{2}{*}{ No Data } & \multirow[t]{2}{*}{+50} & $+198 /+114$ & -33 \\
\hline & & & & $+198 /+154$ & -25 \\
\hline
\end{tabular}

effects. The IONICS 3Q 120 mass spectrometer detector with its fast polarity switching capabilities facilitated the acquisition of accurate, high precision data for atrazine, hydroxyatrazine (ESI positive), and dicamba (ESI negative) within one LC run.

\subsection{Calculations}

Equation (2) can be rearranged to give

$$
\frac{\theta_{1}}{M_{1}}=-K_{e q} \theta_{1}+K_{e q} \theta
$$

Thus, sorption capacities $(\theta)$ for each pesticide can be calculated by plotting $\theta_{1} / M_{1}$ against the equilibrium uptake $\theta_{1}$ for each equilibrated soil-solution mixture for each pesticide. This gives a slope of $-K_{e q}$ and an extrapolated intercept of $K_{e q}$ multiplied by the sorption capacity $\theta$. This technique is most reliable when the uptake approaches saturation, which reduces the uncertainty involved in the extrapolation to the intercept.

The chemical requirements for complete kinetic modeling similarly require determining numbers of empty and filled surface sorption sites, solution concentrations of a pesticide and its reaction products, and the amount of pesticide irreversibly trapped by soil particles. All of these variables are time dependent. Time dependent measurements to elucidate the details of the uptake kinetics were not explicitly performed as part of the current study, but are an important part of the complete picture. These procedures, and results for selected systems, are more fully described elsewhere [1]-[6].

In summary, multiple fates are possible for each pesticide. Current experimental techniques make it possible to measure or infer each fraction. Residual pesticides remaining in solution, as well as any decomposition byproducts which appear in solution, can be measured directly by LC-MS/MS. Pesticides which loosely sorb onto the surface of the soil can be measured by injection of a soil/solution slurry, as described in [1]-[6]. This gives a measure of the labile (easily recovered) fractions. The remainder has either volatolized, or diffused more deeply into the soil particles to become more tightly bound (sequestered via diffusion into pores). In the current treatment, losses due to volatilization are assumed to be negligible. The amount of each pesticide remaining sequestered within the soil can therefore be inferred by mass balance. Complete kinetic update data acquired using these techniques, including predictive calculations, have been presented and described more fully elsewhere [1]-[6].

In the current study, recovery of pesticide residues from previously exposed soil samples was examined by direct measurement of solution concentrations after desorption times of one and eleven days. Some of the material thus recovered, especially after eleven days, could include material which has slowly diffused back out of the pores. Measurements like this have implications for sequestration and long range transport, and demonstrate the importance of distinguishing between labile and more tightly bound fractions.

\section{Results}

Using the conditions described, atrazine, hydroxyatrazine and dicamba eluted from the column within four minutes, giving well resolved peaks with good shape (Figure 2). Calibration curves for the quantifiers showed 


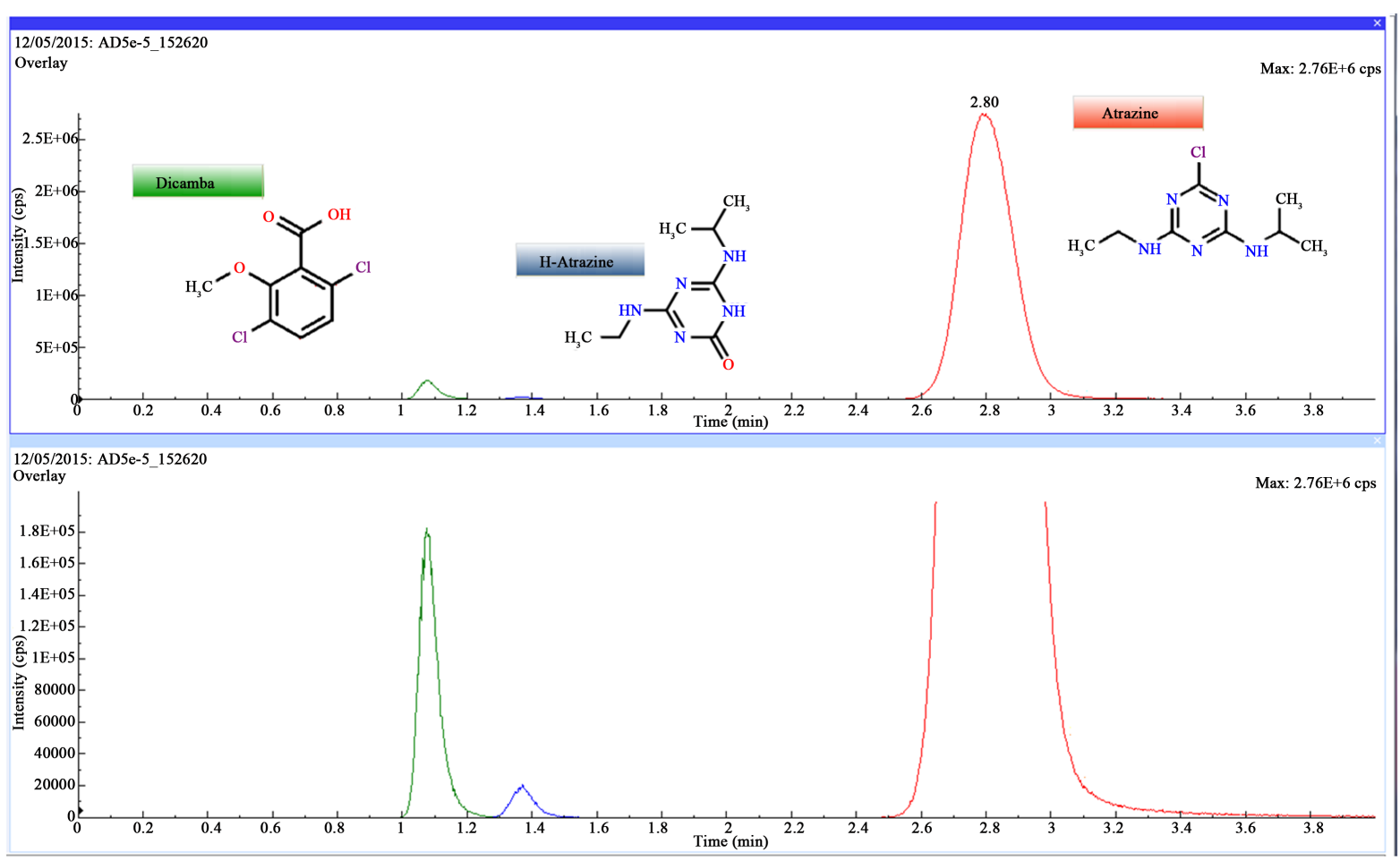

Figure 2. LC Chromatogram showing elution of atrazine, dicamba, and hydroxyatrazine within four minutes with good separation and peak shape. The chemical structure drawings were acquired from ChemSpider.

good linearity over the entire dynamic range (Figure 3). These calibration curves were used to determine solution concentrations for the equilibrated soil-pesticide mixtures for the entire range of samples. Pesticide uptake by the soil aliquots was calculated by difference, between the known initial concentrations and the amounts remaining in solution at equilibrium. Ideally saturation should be approached for the higher initial concentrations of pesticides. An approach to saturation is demonstrated in Figure 4, as the uptake begins to plateau at higher concentrations.

Using these data and plotting $\left(\theta_{1} / M_{1}\right)$ vs. $\theta_{1}$, it is possible to extrapolate to determine a value for $\theta$, the maximum sorption capacity for a given pesticide on that soil. Extrapolating in this fashion resulted in sorption capacities, $\theta$, of $0.051 \mathrm{mmol} / \mathrm{g}$ for atrazine and $0.070 \mathrm{mmol} / \mathrm{g}$ for dicamba. These numbers have taken into account ionization efficiency effects in the ion source, by using water which was previously exposed to soil for preparing the calibration standards. Adopting this technique produced observable differences in the calibration curves when compared with standards prepared with ultra-pure, HPLC grade water, and allowed us to avoid the use of expensive, deuterated internal standards.

The sorbed pesticides are known to partition into labile (easily recovered) and more tightly bound fractions. Desorption experiments were conducted to explore the desorption processes. Small but easily measurable quantities of both atrazine and dicamba were recovered after exposure of the doped soil aliquot to fresh water for one day. In the case of atrazine, a small percentage of the decomposition byproduct, hydroxyatrazine, was also recovered. The experiment was repeated for recovery after eleven days. In the case of dicamba, no clear increase was seen in the amount desorbed (recovered) after eleven days vs. one. For atrazine, recovery was greater for both the atrazine and hydroxyatrazine after eleven days. Partitioning of the atrazine and dicamba after one and eleven days are listed in Table 2 and Table 3.

\section{Discussion}

The results presented above demonstrate the types of experiments which can be conducted to determine the fate of pesticides interacting with soils, and the accuracy, precision, and completeness of data which can be acquired using LC-MS/MS. Another advantage of MS/MS detection is the ability to simultaneously quantify numerous pesticides and decomposition byproducts within one LC run. The fast polarity switching capability of the $3 \mathrm{Q}$ 


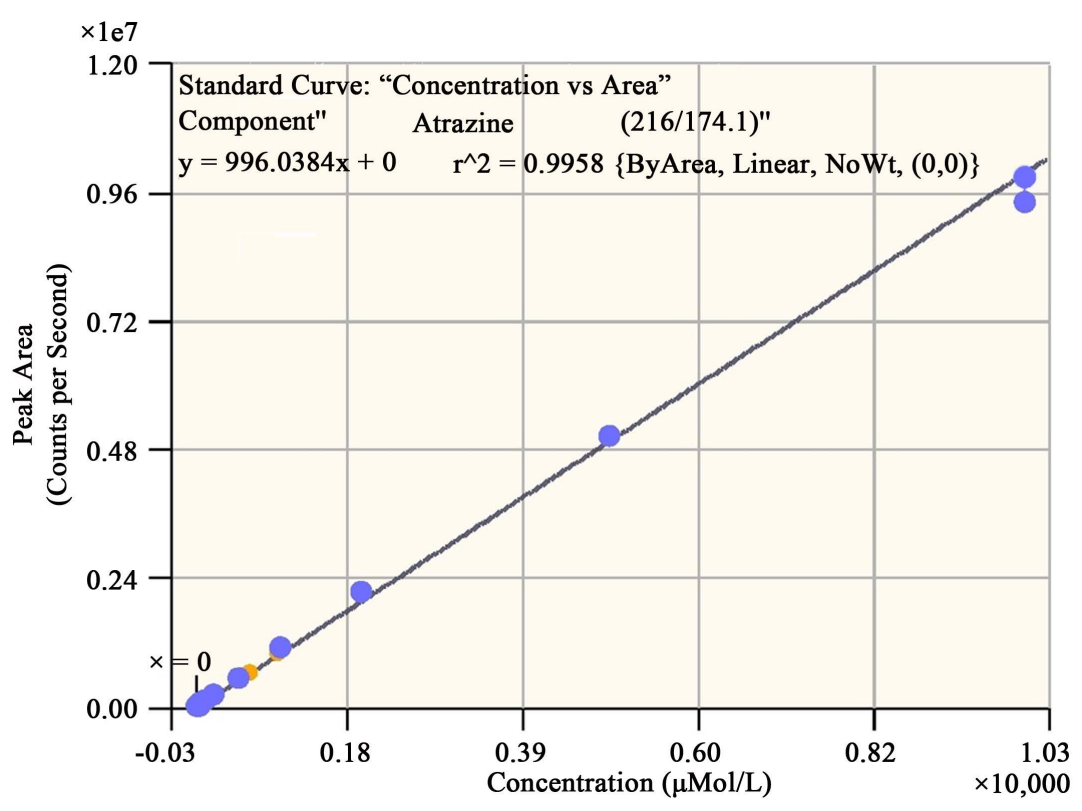

(a)

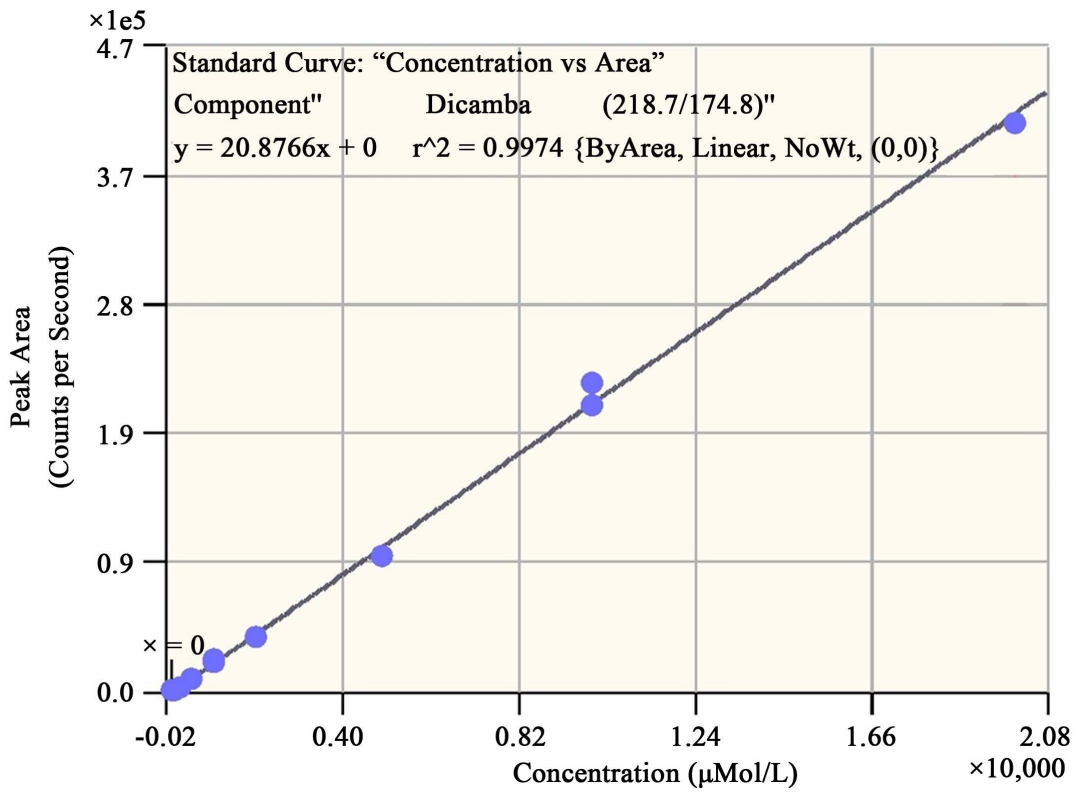

(b)

Figure 3. Calibration curves for atrazine (a) and dicamba (b) demonstrating good linearity across the entire dynamic range.

Table 2. Partitioning of atrazine after 1 and 11 days, showing recovery of sorbed material.

\begin{tabular}{ccc}
\hline Atrazine & 1 day & 11 days \\
\hline Atrazine in solution (\%) & $89.8 \pm 0.05$ & $89.8 \pm 0.05$ \\
H-Atrazine in solution (\%) & $1.75 \pm 0.05$ & $1.75 \pm 0.05$ \\
Atrazine labile (\%) & $0.87 \pm 0.03$ & $1.36 \pm 0.03$ \\
H-Atrazine labile (\%) & $0.06 \pm 0.02$ & $0.11 \pm 0.02$ \\
Bound (\%) & $7.49 \pm 0.15$ & $6.95 \pm 0.15$ \\
\hline
\end{tabular}




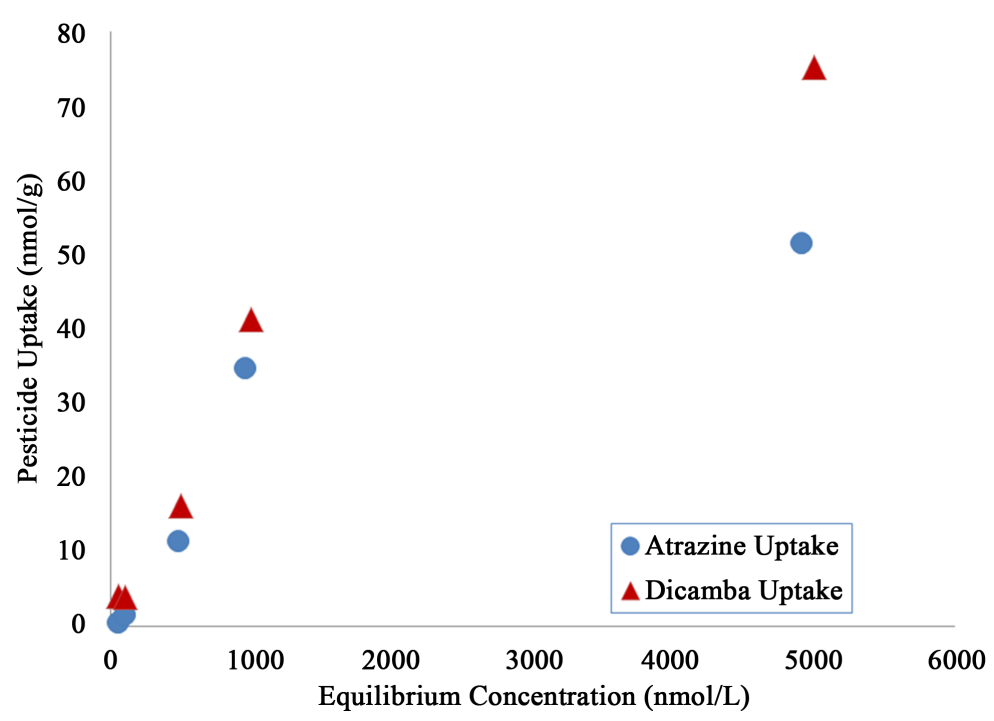

Figure 4. Uptake of atrazine and dicamba by soil. Uptake begins to plateau at higher concentrations, indicating the approach of saturation.

Table 3. Partitioning of dicamba after 1 and 11 days, showing recovery of sorbed material.

\begin{tabular}{ccc}
\hline Dicamba & 1 day & 11 days \\
\hline In solution (\%) & $86.1 \pm 0.05$ & $86.1 \pm 0.05$ \\
Labile (\%) & $0.12 \pm 0.02$ & $0.23 \pm 0.02$ \\
Bound (\%) & $13.73 \pm 0.07$ & $13.62 \pm 0.07$ \\
\hline
\end{tabular}

120 triple quadrupole mass spectrometer facilitated the measurement of both atrazine, which preferentially forms positive ions, and dicamba, which forms negative ions, within a single experiment.

Extending these types of experiments provides a more complete picture, including the kinetics of sorption and fractionation into labile (easily recovered) fractions. Experiments like this have been conducted for other systems, such as the pesticide chlorothalonil on a Simcoe soil (Figure 5) [10].

Data such as these can be used to determine sorption-desorption rate coefficients which can be used for predictive calculations which are more accurate than calculations conducted using more conventional parameter such as partition coefficients (Figure 6). Using partition coefficients instead of fundamental kinetic rate coefficients results in an initial trend for solution concentration which is completely reversed (increasing rather than decreasing with time). In addition, depending on which partition coefficient is used, the predicted solution concentration can be off by as much as a factor of five or six. These discrepancies are obviously significant, and demonstrate the dangers of attempting to predict pesticide behavior using partition coefficient data. Experimentally measuring the fundamental rate coefficients clearly gives much better, and much more correct data for predicting solution concentrations over time.

In the forensic science context, an advantage of this approach is that everything is accounted for. That includes the changes with time of all of the physical and chemical forms of the toxins. Ideally, these accurate, predictive chemical models would be integrated into existing, high quality hydrology models such as the US-EPA's Pesticide Root Zone Model for Ground Water (PRZM GW) model. The ability of such a model to combine accurate predictions on the movement of surface or groundwater with accurate chemical information would prove invaluable for use in environmental assessments, remediation, and litigation.

\section{Summary and Conclusion}

The data and experiments presented here represent a sample of the kind and quality of data which can be used to construct chemically accurate, predictive models for pesticide solution concentrations and soil interactions in the 


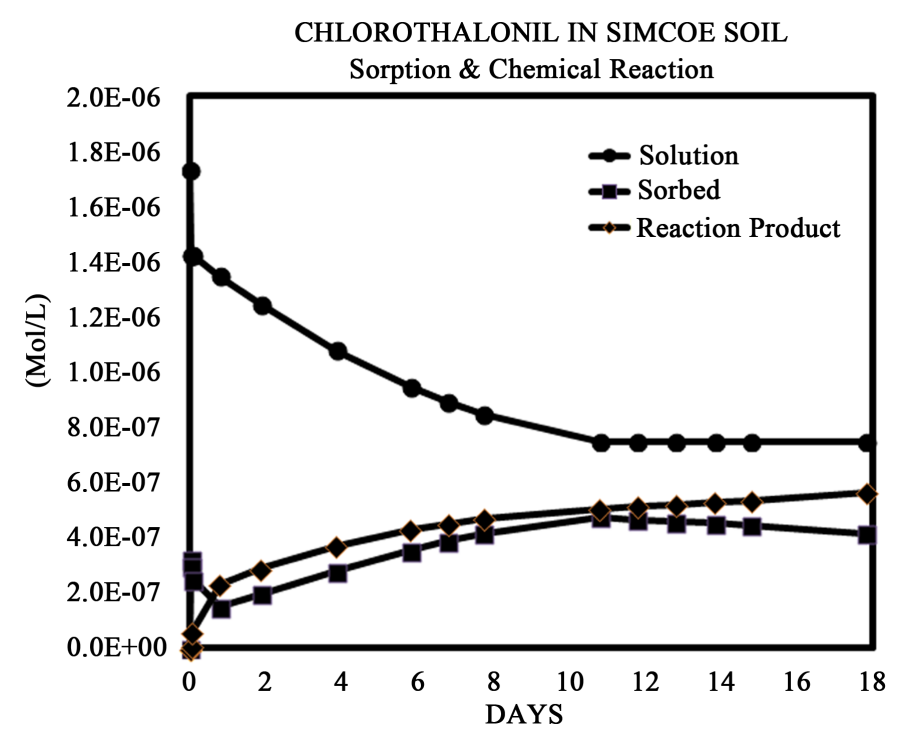

Figure 5. Detailed reaction kinetics showing the uptake of chlorothalonil by a Simcoe soil, showing partitioning into solution phase residues, easily recovered (labile sorbed) fractions, and decomposition into a reaction product or products.

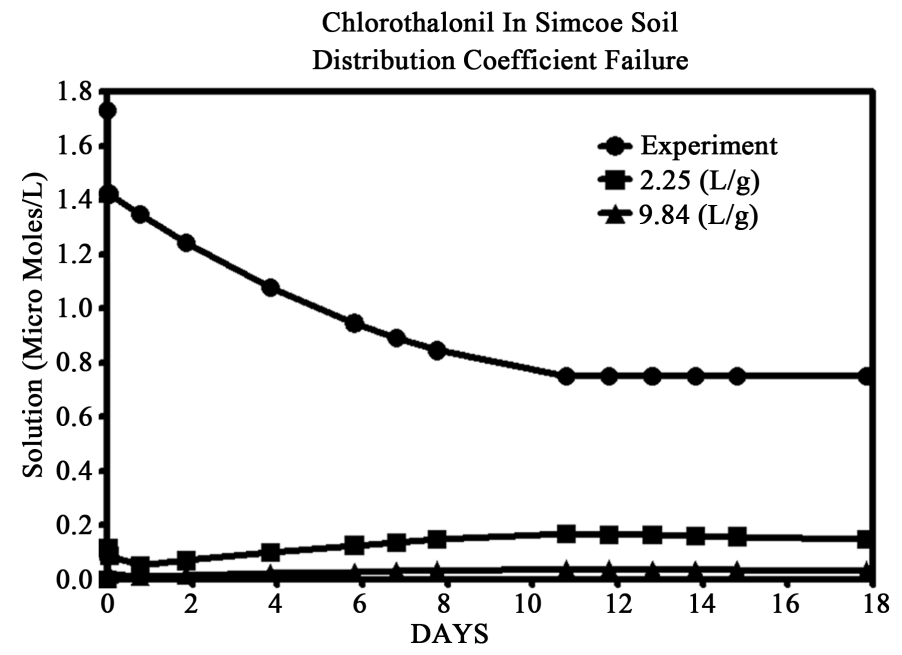

Figure 6. Tests of the distribution coefficient KD with previous experimental data for Chlorothalonil in Simcoe Soil [10]. KD values are shown for 0.78 and 7.7 days.

environment. LC-MS/MS provides a suitable technique for efficiently acquiring large quantities of good quality data which can be used to calculate fundamental kinetic rate coefficients for the interactions of pesticides with soils and soil components. These data have been incorporated into predictive kinetic models, which accurately and successfully reproduce the pesticide solution concentrations over time. Integration of such data and predictive models with hydrology models would provide an extremely valuable tool for the Environmental Forensics community.

\section{References}

[1] Gamble, D.S. (2013) Discoveries Leading to Conventional Chemical Kinetics for Pesticides in Soils: A Review. In: Sparks, D.L., Ed., Advances in Agronomy, Vol. 120, Academic Press, Elsevier Inc., 381-420. http://dx.doi.org/10.1016/b978-0-12-407686-0.00006-3

[2] Gamble, D.S., Webster, G.R.B. and Lamoureux, M. (2012) Propanil in a Manitoba Soil: An Interactive Spreadsheet Model Based on Conventional Chemical Kinetics. Journal of Environmental Monitoring, 14, 1167-1173. 
http://dx.doi.org/10.1039/c2em10366d

[3] Gamble, D.S. (2008) Atrazine Sorption Kinetics in a Characterized Soil: Predictive Calculations. Environmental Science and Technology, 42, 1537-1541. http://dx.doi.org/10.1021/es702432v

[4] Gamble, D.S. (2009) Herbicide Sorption by Immersed Soils: Stoichiometry and the Law of Mass Action in Support of Predictive Kinetics. Environmental Science and Technology, 43, 1930-1934. http://dx.doi.org/10.1021/es8025177

[5] Gamble, D.S., Webster, G.R.B. and Lamoureux, M. (2010) Quantitative Prediction of Atrazine Sorption in a Manitoba Soil Using Conventional Chemical Kinetics instead of Empirical Parameters. Journal of Physical Chemistry C, 114, 20055-20061. http://dx.doi.org/10.1021/jp1063929

[6] Gamble, D.S., Webster, G.R.B. and Lamoureux, M. (2012) Pesticide Reaction Mechanisms in Soil: An Interactive Spreadsheet Model Based on Conventional Chemical Kinetics. Journal of Environmental Monitoring, 14, 1166-1172. http://dx.doi.org/10.1039/c2em10366d

[7] Sitea, A.D. (2001) Factors Affecting Sorption of Organic Compounds in Natural Sorbent Water Systems and Sorption Coefficients for Selected Pollutants: A Review. Journal of Physical and Chemical Reference Data, 30, 187. http://dx.doi.org/10.1063/1.1347984

[8] Wauchope, R.D., Yeh, S., Linders, J.B.H.J., Kloskowski, R., Tanaka, K., Rubin, B., Katayama, A., Kördel, W., Gerstl, Z., Lane, M. and Unsworth, J.B. (2002) Pesticide Soil Sorption Parameters: Theory, Measurement, Uses, Limitations and Reliability. Pest Management Science, 58, 419-445. http://dx.doi.org/10.1002/ps.489

[9] Baris, R., Barrett, M., Bohaty, R., Echeverria, M., Kennedy, I., Malis, G., Wolf, J. and Young, D. (2012) Final Report: Identification and Evaluation of Existing Models for Estimating Environmental Pesticide Transport to Groundwater. Health Canada, U.S. Environmental Protection Agency. http://www.epa.gov/oppefed1/models/water/przm gw/wqtt przm gw guidance.htm\#foot 1

[10] Gamble, D.S. (2015) Pesticides in Soils: Correct Kinetics and Flawed Parameters-More Experimental Evidence. 250th American Chemical Society National Meeting and Exposition, Boston, 16-20 August 2015, Oral Presentation. 